\title{
Content based Image Retrieval using Model Approach
}

\author{
Kunal Shriwas \\ Electronics \&Telecommunication Engg \\ Department \\ St. Francis Institute of Technology \\ Mumbai, India
}

\author{
Vaqar Ansari \\ Electronics \& Telecommunication Engg \\ Department \\ St. Francis Institute of Technology \\ Mumbai, India
}

\begin{abstract}
Due to rapid development of digital and information technologies, more multimedia information is generated and available in digital form from varieties of resources around the world. Content based image retrieval systems (CBIR) are designed to allow users to search images in large databases which match closely with user's query image. Proposed framework consists of all three features to achieve better retrieval results. The color feature is extracted by quantifying the HSV color space and the color attribute like mean value, standard deviation and the image bitmap of HSV color space. The edge feature are obtained by edge histogram descriptor. Texture features are obtained by entropy based gray level cooccurrence matrix (GLCM). Euclidian distance is used to find similarity measurement between query image and database images.
\end{abstract}

\section{Keywords}

Content based image retrieval (CBIR); HSV; Image binary bitmap; Gray level co-occurrence matrix (GLCM); Edge histogram descriptor (EHD).

\section{INTRODUCTION}

With the revolution of the internet and the availability of image capturing devices such as image scanners, digital camera mobile phones, plenty of images have been produced and stored throughout the world. It is very important to retrieve images for different application such as art galleries, architectural and engineering design, remote sensing and management of earth resources, scientific database management .But to utilize it, an efficient process is required. For this retrieval purpose two general purpose retrieval systems have been developed. They are text-based and content-based retrieval system. In text-based approach images are manually annotated by text descriptors which are then used by database management system (DBMS) to perform retrieval process. But it has leads to two disadvantages. The first one is annotation inaccuracy due to subjectivity of human perception. The second, a considerable level of human labor is required for manual annotation. To overcome such disadvantages in text-based retrieval system, CBIR was introduced.

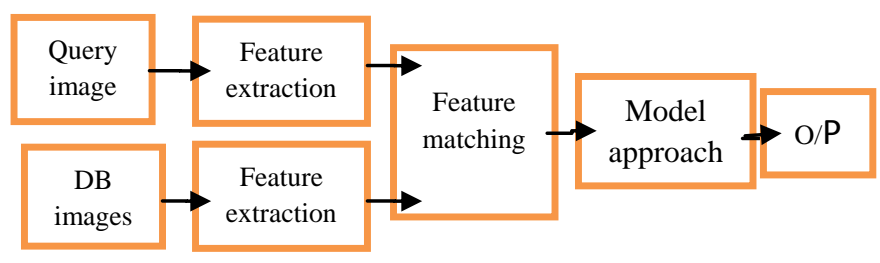

Fig.1 General Block diagram of CBIR [1]

In CBIR, images are ranked by their content, such as color, texture, edge, shapes. CBIR system can be implemented based on single feature. Single feature represents the content of an image from specific angle, it may be suitable for some images, but also may be difficult to describe other images. Therefore representing an image with multi feature from multi-angle is expected to achieve better results.

\section{LITERATURE REVIEW}

Content based image retrieval, a technique which uses visual content to search images from large databases according to user's interests, has been an active research area since the 1990s. Several general purpose systems have been developed. Query by Image Content (QBIC) [2] system was first commercial system for CBIR developed by IBM Almaden Research Center, San Jose in 1995. This system uses color, texture, shape, sketches for image representation. NETRA [3] system has been developed by Department electrical and computer engineering at University of California, Santa Barbara in 1997. It uses Gabor wavelet transform and curvature function of contour to represent texture and shape feature respectively. Key-point Indexing Web Interface (KIWI) [4], has been developed in France by INSA Lyon in 2001. This system extracts the key points in the query image instead of entire image. Photobook [5] was developed by Vision and Modelling Group at MIT Media Lab in 1997. Photobook consists of three sub-books. They are Appearance Photobook, Shape Photobook and Texture Photobook. Which can extract the face, shape, texture respectively.

Image Miner [6] has been developed by Technology-Zentrum Informatics at University of Bremen in Germany in 1997. For similarity measurement special module is developed within the system. Chin-Chin Lai et.al. [7] have proposed an interactive genetic algorithm (IGA) to reduce the gap between the retrieval's results and the user's expectations. Meenakshi Madugunki et.al. [8] have explained detailed classification of CBIR systems. They used color histogram for color, discrete wavelet transform (DWT) for texture feature. Nhu-Van Nguyen et.al [9] have proposed clustering and image mining technique. Same technique is used by A. Kannan et.al [10], the main objective is to remove the data loss and extract useful information. Zhang XU-bo et.al. [11] have proposed improved K-means clustering and relevance feedback. sharadh Ramaswamy et.al. [12] have proposed method on fast clustering-based indexing technique. OLIVE (2008) provides dual access to web images and PIRIA visual search engines. Selection of feature is one of the important aspects of image.

\section{IMAGE FEATURE}

\subsection{Color Feature}

Color is most used visual content for image retrieval. A color image can be represented using three primaries of color space. But the RGB space does not correspond to the human way of perceiving the colors and does not separate luminance component from chrominance ones therefore we use HSV color space in our approach. HSV is an intuitive color space in 
the sense that each component corresponds directly to visual perception. Hue is used to distinguish colors. Saturation gives measure of the percentage of white light added to a pure color. Values gives the amount of intensity. The color distribution of pixels in an image contains sufficient information. The mean of the pixel colors states color of the image and standard deviation can represent variation of a pixel colors. We can use this two features to represent properties of an image. The mean and standard deviation can be defined as follows:

$\mu=\frac{1}{N} \sum_{i=1}^{N} P_{i}$

$\sigma=\left[\frac{1}{N-1} \sum_{i=1}^{N}\left(P_{i}-\mu\right)^{2}\right]^{\frac{1}{2}}$

where $\mu=[\mu H, \mu S, \mu V]^{T}$ and $\sigma=[\sigma H, \sigma S, \sigma V]^{T}$,each component $\mu$ and $\sigma$ indicates HSV information,

respectively,$P_{i}$ indicates the $i$ th pixel of an image. Results are shown in table number 4 .

\subsection{Texture Feature}

The texture is another type of useful information for image retrieval. Texture provides the surface properties of an image and their relationship with surrounding environment. We use gray level co-occurrence matrix (GLCM). GLCM creates a matrix with the directions and distances between pixels, and then extract meaningful statistics from the matrix as a texture feature. GLCM Is composed of probability value $p(i, j ; d, \theta)$ which is defined by following equation:

$p(i, j ; d, \theta)=\#\left\{\left(x_{1}, y_{1}\right)\left(\left(x_{2}, y_{2}\right) \mid g\left(x_{1}, y_{1}\right) i, g\left(x_{2}, y_{2}\right)=\mathrm{j}\right.\right.$,

$\left.\left|\left(x_{1}, y_{1}\right)-\left(x_{2}, y_{2}\right)\right|=d, \angle\left(\left(x_{1}, y_{1}\right)-\left(x_{2}, y_{2}\right)\right)=\theta\right\}$

where, \#=number of occurrences inside the window, $\mathrm{i}=$ intensity level of first pixel at location $(\mathrm{x} 1, \mathrm{y} 1), \mathrm{j}=$ intensity level of first pixel at location (x2,y2). The entropy (E) is used to capture the textural information in an image and it is defined by following formula:

$$
E=-\sum_{i, j} C_{i, j} \log C_{i, j}
$$

where, $C i, j=\mathrm{GLCM}$,

Entropy gives a measure of complexity of an image. Retrieval results based on texture feature is tabulated below in table number 5 .

\subsection{Edge Feature}

Edges in images consist of an important feature to represent their content. One way of representing such an important feature is to use a histogram. An edge histogram represents the frequency and directionality of brightness changes in the image. Edge histogram descriptor (EHD) is used to extract edge feature of an image. The EHD is basically the distributions of 5 types of edges in its each sub-image. The sub-image is obtained by dividing the image space into $4 * 4$ non-overlapping blocks. We then generate a histogram of edge distribution for each sub image. Edges in the sub-image are categorized into five types: vertical, horizontal, 45-degree diagonal, 135-degree diagonal, and non-directional edges. So the histogram for each sub-image represents the relative frequency of occurrences of the five types of edges in the corresponding sub-image. Each histogram bin values are normalized and quantized. For normalization, the number of edge occurrences for each bin is divided by total number of image blocks in the sub-image. Results using edge feature is mentioned in table number 6 .

\section{SIMILARITY MATCHING}

The commonly used similarity measure method is the distance method. There are various distance methods available such as Euclidian distance, City Block distance and Canberra distance. Initially three features of a query image i.e. color, texture, edge are extracted by their respective methods. Same features of the database images are extracted and stored. We use Euclidian distance method for the similarity measurement between query image and database images.

\section{MODEL APPROACH}

This is the novel approach which can be explained in following steps:

1) Extract the image features of the database image

2) Retrieve the images based on individual features by comparing.

3) Store all the results obtained from above step in one matrix.

\begin{tabular}{|l|l|l|l|l|l|}
\hline Feature & 1 & 2 & 3 & 4 & 5 \\
\hline Edge & a & b & c & d & e \\
\hline Texture & a & f & c & g & e \\
\hline Color & c & d & e & f & a \\
\hline
\end{tabular}

4) Perform zigzag scanning as shown in table number 1. And we will get 3 columned table. In which first Column represent image, second represent number of time it occurs during zigzag scanning, third column represent weights.

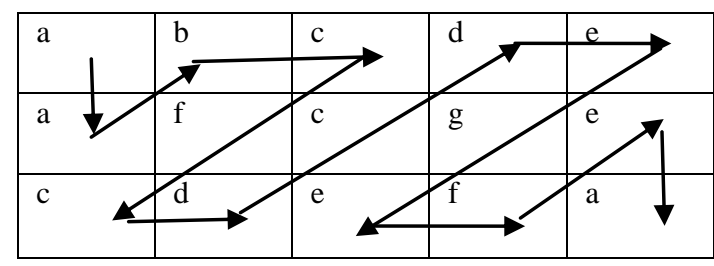

5) Then arrange third column of table in descending order. Corresponding row of first and second column should be shifted according to third column as shown in table number 2 and 3. This model approach can be explained with an example as following:

Table 1: Performing zigzag scanning

\begin{tabular}{|c|c|c|}
\hline Image & $\begin{array}{c}\text { Number of times it } \\
\text { occurs }\end{array}$ & Weights \\
\hline $\mathrm{a}$ & 1 & 15 \\
\hline $\mathrm{a}$ & 1 & 14 \\
\hline $\mathrm{b}$ & 1 & 13 \\
\hline $\mathrm{c}$ & 1 & 12 \\
\hline
\end{tabular}




\begin{tabular}{|c|c|c|}
\hline f & 1 & 11 \\
\hline $\mathrm{c}$ & 1 & 10 \\
\hline $\mathrm{d}$ & 1 & 9 \\
\hline $\mathrm{c}$ & 1 & 8 \\
\hline $\mathrm{a}$ & 1 & 1 \\
\hline $\mathrm{d}$ & 1 & 6 \\
\hline $\mathrm{e}$ & 1 & 5 \\
\hline $\mathrm{g}$ & 1 & 4 \\
\hline $\mathrm{e}$ & 1 & 3 \\
\hline $\mathrm{f}$ & 1 & 2 \\
\hline $\mathrm{e}$ & 1 & 1 \\
\hline $\mathrm{a}$ & & \\
\hline
\end{tabular}

Table 2: Repetitive images

\begin{tabular}{|c|c|c|}
\hline Image & $\begin{array}{c}\text { Number of times it } \\
\text { occurs }\end{array}$ & Weights \\
\hline $\mathrm{a}$ & 3 & 20 \\
\hline $\mathrm{b}$ & 1 & 13 \\
\hline $\mathrm{c}$ & 3 & 16 \\
\hline $\mathrm{d}$ & 2 & 12 \\
\hline $\mathrm{e}$ & 3 & 3 \\
\hline $\mathrm{f}$ & 1 & 5 \\
\hline $\mathrm{g}$ & 1 & \\
\hline
\end{tabular}

Table 3: Adding weights of repeated image

\begin{tabular}{|c|c|c|}
\hline Image & $\begin{array}{c}\text { Number of times it } \\
\text { occurs }\end{array}$ & Weights \\
\hline $\mathrm{c}$ & 3 & 28 \\
\hline $\mathrm{a}$ & 3 & 16 \\
\hline $\mathrm{d}$ & 2 & 13 \\
\hline $\mathrm{b}$ & 1 & 12 \\
\hline $\mathrm{e}$ & 3 & 5 \\
\hline $\mathrm{g}$ & 1 & 3 \\
\hline $\mathrm{f}$ & 1 & \\
\hline
\end{tabular}

As shown in previous table proposed method gives out most relevant images, out of that we select top 5 images as an output.

\section{EXPERIMENT AND ANALYSIS}

In this paper experimental data set contains 1000 images, divided into 10 categories, each category has 100 images.

Experimental images covers variety of content, including people, beach, buildings, buses, dinosaurs, elephant, flower, horses, mountains, food. Precision and recall rate are used to evaluate the performance of above approach. We selected top 10 images. And calculated precision and recall by using following formula.

Precision $=$ Number of relevant images similar to query $/$ Number of images retrieved

Recall $=$ Number of relevant images similar to query/ total number of relevant images available in the database.

For comparison initially we found results based on single feature. And then we combine all three features and evaluated precision and recall values. They are as follows:

Table 4: Results using color feature:

\begin{tabular}{|l|c|c|}
\hline Category & $\begin{array}{l}\text { Precision } \\
\text { values (\%) }\end{array}$ & $\begin{array}{l}\text { Recall } \\
\text { values (\%) }\end{array}$ \\
\hline Africa people \& village & 70 & 7 \\
\hline Beach & 50 & 5 \\
\hline Buildings & 70 & 7 \\
\hline Buses & 100 & 10 \\
\hline Dinosaurs & 100 & 10 \\
\hline Elephant & 90 & 9 \\
\hline Flower & 60 & 6 \\
\hline Horses & 100 & 10 \\
\hline Mountains \& glaciers & 90 & 9 \\
\hline Food & 80 & 8 \\
\hline Average & & 8.1 \\
\hline
\end{tabular}

Table 5: Results using Texture feature:

\begin{tabular}{|l|c|c|}
\hline Category & $\begin{array}{l}\text { Precision } \\
\text { values (\%) }\end{array}$ & $\begin{array}{l}\text { Recall } \\
\text { values (\%) }\end{array}$ \\
\hline Africa people \& village & 20 & 2 \\
\hline Beach & 40 & 4 \\
\hline Buildings & 80 & 8 \\
\hline Buses & 100 & 10 \\
\hline Dinosaurs & 100 & 10 \\
\hline Elephant & 60 & 6 \\
\hline
\end{tabular}


International Journal of Applied Information Systems (IJAIS) - ISSN : 2249-0868

Foundation of Computer Science FCS, New York, USA

Volume 10-No.8, April 2016 - www.ijais.org

\begin{tabular}{|l|c|c|}
\hline Flower & 100 & 10 \\
\hline Horses & 100 & 10 \\
\hline Mountains \& glaciers & 40 & 4 \\
\hline Food & 60 & 6 \\
\hline Average & 70 & 7 \\
\hline
\end{tabular}

Table 6: Results using Edge feature:

\begin{tabular}{|l|c|c|}
\hline Category & $\begin{array}{l}\text { Precision } \\
\text { values (\%) }\end{array}$ & $\begin{array}{l}\text { Recall } \\
\text { values (\%) }\end{array}$ \\
\hline Africa people \& village & 30 & 3 \\
\hline Beach & 30 & 3 \\
\hline Buildings & 30 & 3 \\
\hline Buses & 10 & 1 \\
\hline Dinosaurs & 90 & 9 \\
\hline Elephant & 10 & 1 \\
\hline Flower & 70 & 7 \\
\hline Horses & 20 & 2 \\
\hline Mountains \& glaciers & 10 & 1 \\
\hline Food & 10 & 1 \\
\hline Average & & 3.1 \\
\hline
\end{tabular}

Table 7: Results using all three features

\begin{tabular}{|l|c|c|}
\hline Category & $\begin{array}{l}\text { Precision } \\
\text { values (\%) }\end{array}$ & $\begin{array}{l}\text { Recall } \\
\text { values (\%) }\end{array}$ \\
\hline Africa people \& village & 70 & 7 \\
\hline Beach & 70 & 7 \\
\hline Buildings & 40 & 4 \\
\hline Buses & 100 & 10 \\
\hline Dinosaurs & 100 & 10 \\
\hline Elephant & 80 & 8 \\
\hline Flower & 70 & 7 \\
\hline Horses & 100 & 10 \\
\hline
\end{tabular}

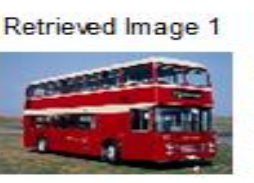

Retrieved Image 4
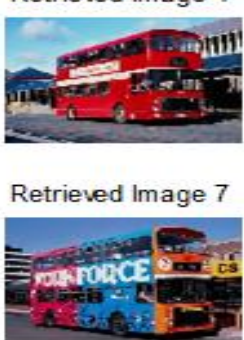

Retrieved Image 10

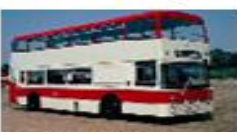

Fig 2: Bus image as a query

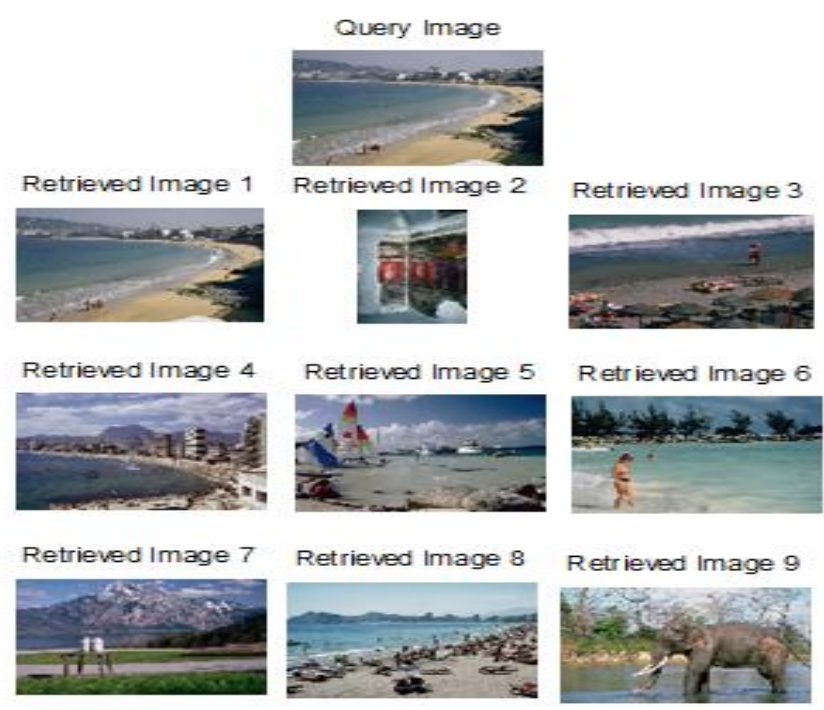

Retrieved Image 10

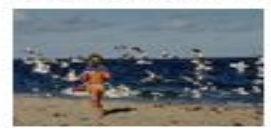

Fig 3: Beach image as a query
Retrieved Im age 3
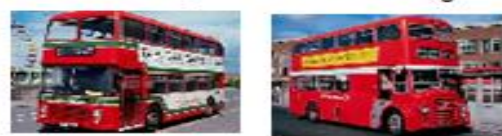

Retrieved Image 6

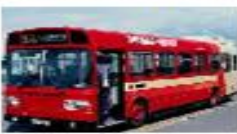

Retrieved Image 9
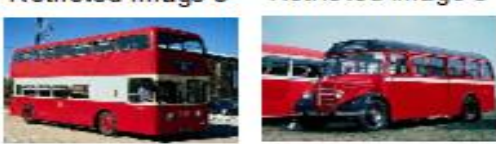

Retrieved Image 3

Retrieved Image 6

Retrieved Image 9 
International Journal of Applied Information Systems (IJAIS) - ISSN : 2249-0868

Foundation of Computer Science FCS, New York, USA

Volume 10-No.8, April 2016-www.ijais.org

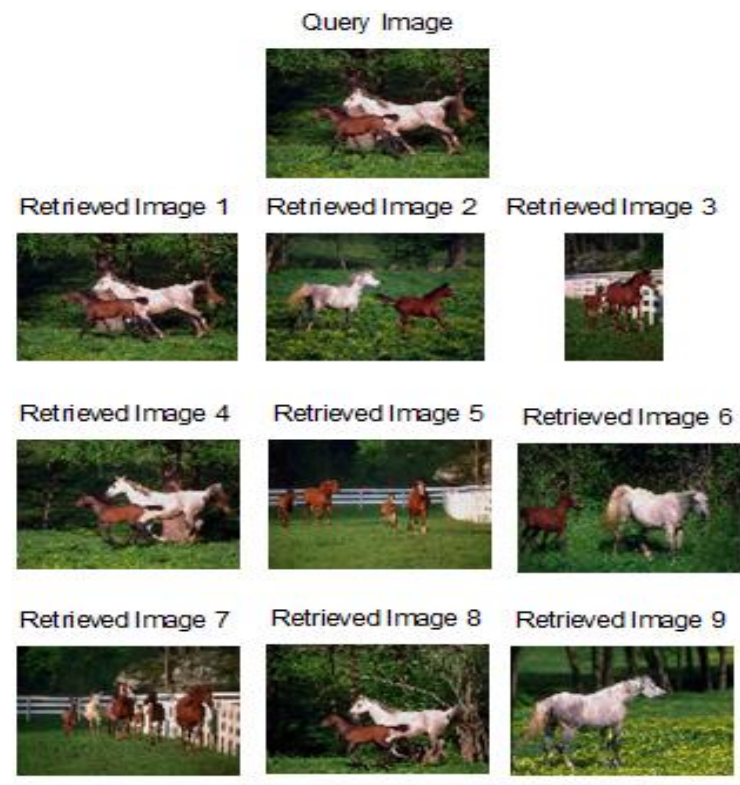

Retrieved Image 10

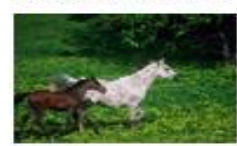

Fig 4: Horses image as a query

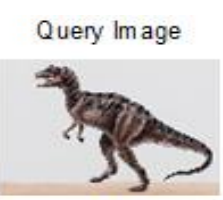

Retrieved Image 1

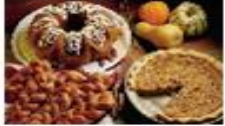

Retrieved Image 2 Retrieved Image 3

Retrieved Image 4
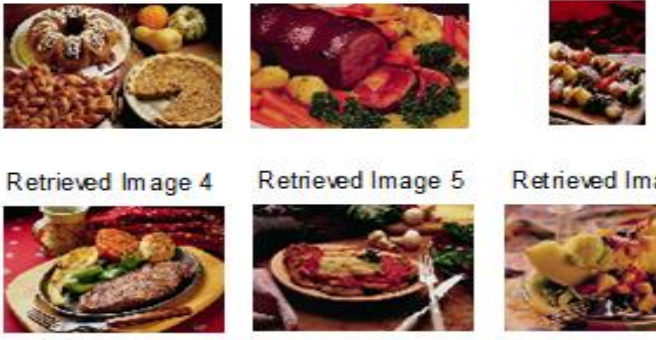

Retrieved Image 5

Retrieved Image 6

Retrieved Image 7
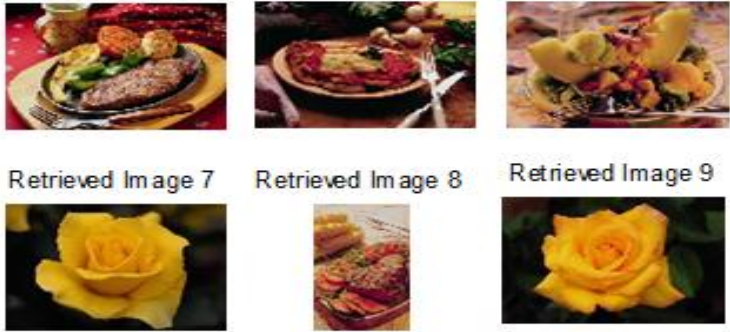

Retrieved Image 9

Retrieved Image 10

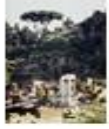

Fig 6: Food image as a query

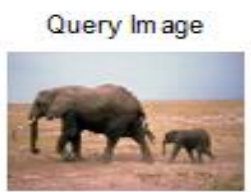

Retrieved Image 1

Retrieved Image 2
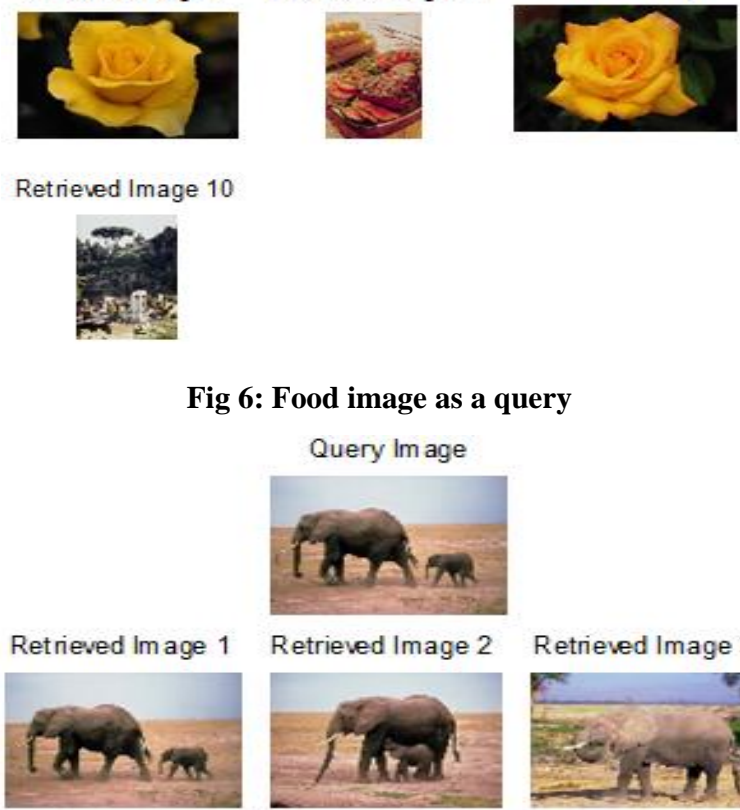

Retrieved Image 3
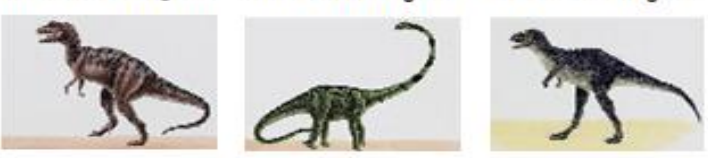

Retrieved Image 4

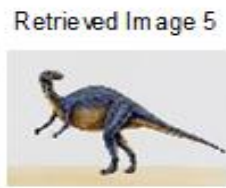

Retrieved Image 6
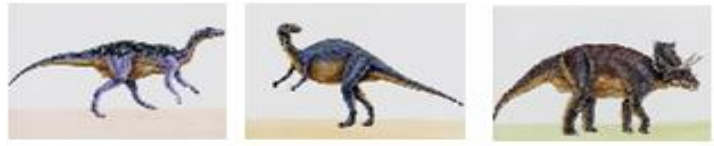

Retrieved Image 7

Retrieved Im age 8
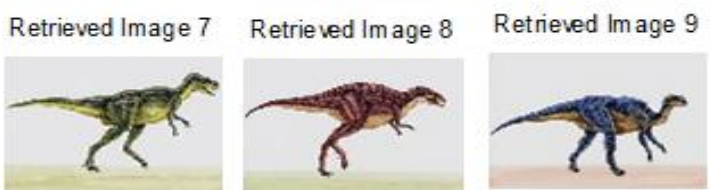

Retrieved Image 10

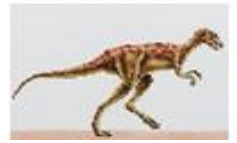

Fig 5: Dinosaur image as a query

\section{Retrieved Im age 4}

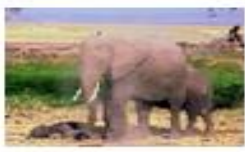

Retrieved Image 5

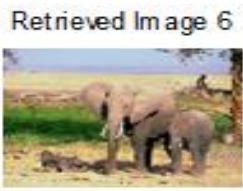

Retrieved Image 7
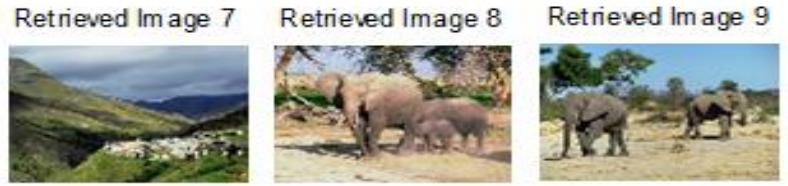

Retrieved Image 10

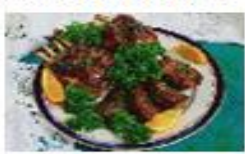

Fig 7: Elephant image as a query 


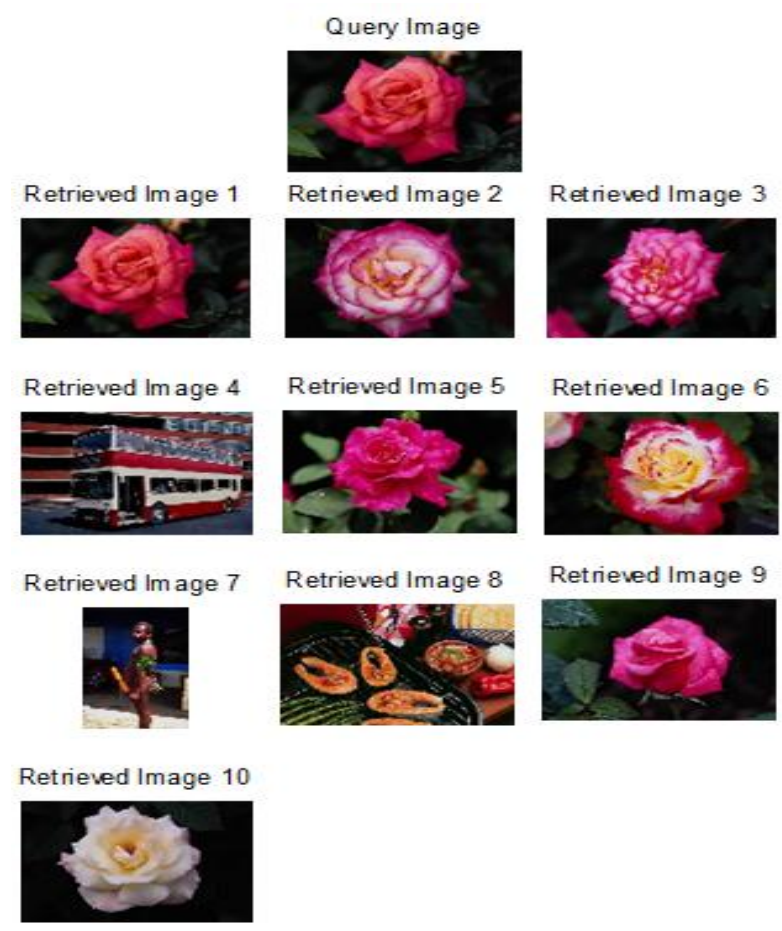

Fig 8: Flower image as a query

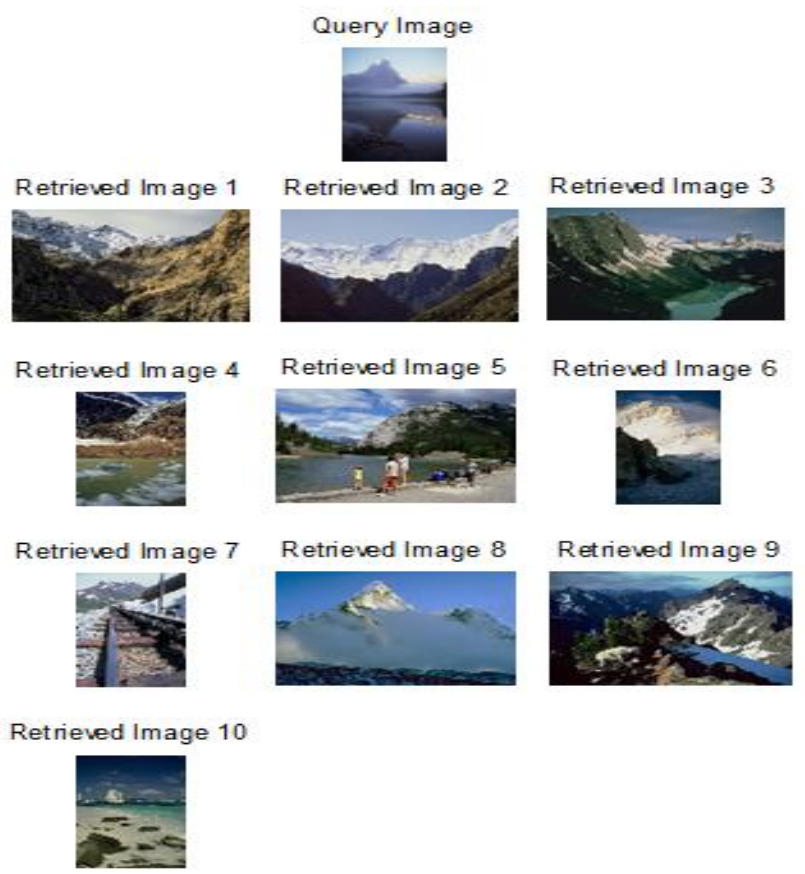

Fig 9: Mountain image as a query

\section{CONCLUSION}

As shown in above results, using single feature for retrieving images from database leads to less precision and recall rate. To improve retrieval efficiency proposed method include three features i.e. color, texture and edge. For the better performance model approach is introduced. According to this approach relevant images can be obtained depending upon their weights. Better results can be obtained by using multi feature combining method and model approach.

\section{REFERENCES}

[1] A.Chadha, S.Mallik, and R.Johar. "Comparative Study and Optimization of Feature-Extraction Techniques for Content based Image Retrieval," International Journal of Computer Applications, vol.52, no.20, 2012.

[2] M. Flickner, H. Sawney, W. Nilback, J. Ashley, Q. Huang, B. Dom, M. Gorkani, J. Halfner, D. Lee, D. petkovic, and p.Yanker, "Query by image and video content: The QBIC system," IEEE Computer vol. 28, no 9, pp.23-32, Sep. 1995.

[3] W. Ma and B.Manjunath, "Natra: A Toolbox for Navigation Large Image Databases," proceedings IEEE Int'l Conf. Image Processing, Santa Barbara, pp.568-571, 1997.

[4] E Loupias and S. Bres, "Key point-Based Indexing for Pre-Attentive Similarities: The Kiwi System," Pattern Analysis and Applications, vol.4, pp.200-214, 2001.

[5] A. Pentland, R.Picard, and S.Sclaroff, "Photobook: Content based manipulation of image databases," International Journal of Computer Vision, vol.18, no 3, pp.233-254, June 1997.

[6] J. Kreyss, M. Roper,P. Alshuth, Th. Hermes, and O. Herzog, "Video Retrieval by Still Image Analysis with Image Minor," Proceedings of IS\&T/SPIE's Symposium on Electronic Imaging: Science \& Technologies, San Jose, CA, 1997.

[7] C.Lai, and Y.Chen. "A user-oriented image retrieval system based on interactive genetic algorithm." IEEE Transactions on Instrumentation and Measurement, vol.60, no. 10, 2011.

[8] D.S.Bormane, M. Madugunki, S. Bhadoria, C. G. Dethe," Comparison of Different CBIR Techniques", IEEE Conference, 2011.

[9] N. V. Nguyen, A. Boucher, J. M. Ogier, S. Tabbone," Clusters- based Relevance Feedback for CBIR: a combination of query movement and query expansion",IEEE Conference, 2010.

[10] A.Kannan, V.Mohan, N.Anbazhagan "Image Clustering and Retrieval using Image Mining Techniques", IEEE Conference, 2010.

[11] Zhangxu-bo," Re-ranking algorithm usingclustering and relevance feedback for image retrieval", IEEE Conference, 2010.

[12] Sharadh Ramaswamy and Kenneth Rose,Fellow, IEEE "Towards Optimal Indexing forRelevance Feedback in Large Image Databases", IEEE transaction on Image Processing, Dec 2009. 